Archived version from NCDOCKS Institutional Repository http://libres.uncg.edu/ir/asu/

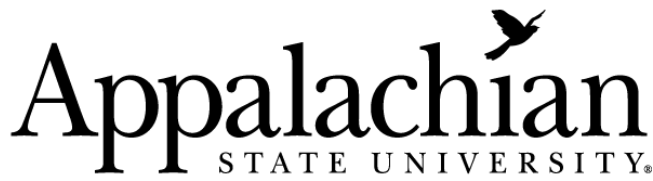

B O O N E, NORT H C A R O L I N A

\section{Retrospective Reports of Child Feeding Practices, Current Eating Behaviors, and BMI in College Students}

\author{
By: M. Martz, Amy T. Galloway \& Claire V, Farrow
}

\begin{abstract}
Research concerning child feeding practices has focused on children and adolescents, and little is known about how feeding practices used in childhood relate to eating behaviors and weight status in early adulthood. We assessed college students' and their parents' retrospective reports of child feeding practices used when the students were in middle childhood. We also assessed the college students' current reports of their eating behaviors using the Dutch Eating Behavior Questionnaire (DEBQ) and the Intuitive Eating Scale (IES), and measured their current BMI. Results showed that college students' and their parents' reports about previous parental use of child feeding practices were not correlated. Parent reports of their own use of child feeding practices were more related to students' eating behaviors and BMI than were students' recollections about feeding practices used by their parents. An analysis of gender effects showed that there were positive correlations between parental child feeding practices, BMI, and emotional eating for female students. These relationships did not exist for male students. The results suggest that child feeding practices recollected by parents are linked to the development of emotional eating and weight status of women in early adulthood.
\end{abstract}

M. Martz, Amy T. Galloway \& Claire V, Farrow (2010) "Retrospective Reports of Child Feeding Practices, Current Eating Behaviors, and BMI in College Students" Volume 18 Issue 7 pp. 1330-1335 Version of Record Available from (www.wileyonlinelibrary.com) DOI: 10.1038/oby.2009.393 


\section{Retrospective Reports of Child Feeding Practices, Current Eating Behaviors, and BMI in College Students}

\section{INTRODUCTION}

Batsell and colleagues (1) reported using retrospective accounts of early feeding interactions to understand food preferences and eating behaviors in adulthood. Findings from this study indicated that $69 \%$ of participants remembered being pressured to eat a disliked food in the past and that $76 \%$ of these occasions involved someone who was an authority figure (e.g., a parent or teacher). Most participants said they still have a strong dislike for foods that they were pressured to eat. These findings raise questions about the significance of everyday feeding interactions between parents and children, and whether these early interactions have a meaningful impact upon eating as children age into adulthood.

Child feeding practices used by parents are related to child weight status and eating behaviors, and some longitudinal and experimental findings suggest that controlling parental feeding practices might influence weight gain and exacerbate problematic eating behaviors in children (2-4). Parents who use controlling feeding practices are theorized to disrupt children's self-regulation of food intake, resulting in over- or undereating (5). Several studies have demonstrated that restrictive feeding practices are related to increases in the likelihood of eating in the absence of hunger (6), whereas pressuring children to eat predicts a decreased preference for pressured food and interferes with food intake in children (7). In one study, 5-year-old girls' perceptions of their parents' use of pressure to eat predicted the girls' restrained and emotional eating (8). There are, however, conflicting findings about the relationship between child feeding practices and child weight status $(9,10)$ that are most likely due to methodological issues, sampling, and differences in the operationalization of constructs.

Although research on this topic has focused predominantly on early and middle childhood, more recent studies have included adolescent samples. For example, the use of pressure to eat by parents has been linked to adolescent's lower weight status, whereas the use of restrictive parental feeding practices was positively related to heavier body weight (11). In another study, the use of psychological control in general parenting (not specific to feeding) was positively related to emotional eating in adolescents (12). Overall, there is a paucity of information concerning how feeding practices might relate to the later development of eating behaviors and body weight.

Most studies focused on child feeding practices have explored the impact of feeding practices used with daughters 
because of an interest in the emergence of dieting behavior that disproportionately affects young women (13). The few studies that have compared the relationships between parent-child feeding practices, and children's eating behavior and BMI in both male and female children have yielded conflicting results. Research indicates that girls receive higher levels of controlling feeding practices and are rewarded more for eating compared to boys $(14,15)$, and that boys are more likely to receive higher levels of pressure to eat (15). In one study, girls', but not boys', eating in the absence of hunger was linked to maternal restriction (16). However, other research has found that parents do not use different levels of controlling feeding practices for girls compared to boys (17). Moreover, Snoek and colleagues (12) reported that there were no gender differences in the relationships between controlling parenting practices in general and emotional eating in girls compared to boys. Overall, research suggests that parents may adapt their feeding practices depending on the gender of the child; this would not be surprising given that parents are known to use different styles of parenting for sons compared to daughters (18).

The purpose of this study was to investigate the relationships between recollections of previous child feeding practices and college students' current BMI, adaptive, and maladaptive eating behaviors. A further aim was to compare students' and their parents' retrospective reports of their perceptions of the child feeding practices that were used when the students were children. We hypothesized that recollections of more controlling child feeding practices reported by parents and students would be positively correlated with current student BMI and more maladaptive eating behaviors. We also hypothesized that parents' and students' recollections of parental child feeding practices would be positively correlated.

\section{METHODS AND PROCEDURES Participants}

Ninety-eight college students from Appalachian State University in the United States and their parents participated in this study that was approved by the University Institutional Review Board. Students were recruited to the study via the research pool for General Psychology at the university. Students were asked to complete a questionnaire, and parents were invited by mail to complete a questionnaire for the study. Written consent was obtained from all participants. Compensation for students was class research credit, and compensation for parents was the opportunity to win a $\$ 50$ gift certificate to a hardware store.

\section{Procedure}

Students completed questionnaires in a classroom with as many as 40 other students in the same room. Following the completion of questionnaires, participants went to another room to have their heights and weights measured privately in triplicate by a research assistant trained in anthropometric procedures.

\section{Measurements}

Background information. Students provided details about who they lived with as a child and who they live with currently. In the parental questionnaire, parents provided information about their relationship to the student, their education history, ethnicity, age, and their selfreported height and weight.

\section{Recollections of child feeding practices}

Parent retrospective Child Feeding Questionnaire: Parents reported their recollections of their previous use of controlling feeding practices using a retrospective version of the Child Feeding Questionnaire (CFQ; 19). The CFQ was designed to examine parents' reports of feeding practices and concerns about the feeding of their child. We adapted this questionnaire by changing the phrasing of each item from present to past tense and by asking parents to think back to when their children were younger (e.g., 5-10 years old) when answering the questions. Middle childhood was chosen because it represents a transitional period when children begin to have more autonomy concerning their food choices. In addition, college students are more likely to be able to remember their parents' feeding practices from this period compared to early childhood. The subscales included parental use of pressure to eat, restriction over the child's food intake, and monitoring of child eating. The CFQ is scored using a Likert scale with response options ranging from (1) disagree to (5) agree, and with higher scores indicating higher levels of control. These subscales have good psychometric properties that include internal consistency levels ranging from $\alpha=0.70-0.92$ (15). In the current study, the parent retrospective CFQ showed good internal reliability for pressure to eat $(\alpha=0.73)$, restriction $(\alpha=0.83)$, and monitoring $(\alpha=0.88)$.

Student retrospective Child Feeding Questionnaire: This questionnaire was designed to examine college students' recollections of the feeding practices used by their parents when they were younger. The scale is an adapted version of the Child Feeding Questionnaire for Children (CFQC; 8) that was designed to compliment the parental version of the CFQ (19). The CFQC was originally created to assess children's perceptions of the child feeding practices used by their parents, specifically, parental use of pressure to eat and restriction. The CFQC uses a five-point response scale ranging from (1) never to (5) always with higher scores corresponding to higher levels of perceived parental control. We modified the children's questionnaire to use with college students and changed the items to past tense. Students were asked to think back to when they were a child and to complete the questionnaire with the person in mind who was most often responsible for feeding them during this time. Carper et al. (8) reported that the CFQC has predictive validity for restrained eating and emotionally disinhibited eating. Using a similar approach to Carper et al. (8), a monitoring subscale was created based on the original CFQ, items included the following: (i) Did your parent keep track of the sweets (candy, ice cream, cake, pies, and pastries) that you ate? (ii) Did your parent keep track of the snack foods (potato chips, Doritos, cheese puffs) that you ate? (iii) Did your parent keep track of the high-fat foods that you ate? In the current study, the student retrospective CFQ showed good internal reliability for restriction $(\alpha=0.71)$, pressure to eat $(\alpha=0.75)$, and monitoring $(\alpha=0.91)$.

\section{Measures of student eating}

Dutch Eating Behavior Questionnaire (DEBQ): The DEBQ was used to measure the prevalence of maladaptive eating behaviors in the student sample. The DEBQ consists of 33 items that are used to examine levels of restrained eating (10 items assessing intentionally restricting food intake), external eating (10 items assessing eating in the absence of hunger), and emotional eating in adults (13 items assessing eating in response to emotional stimuli such as anger, sadness, or boredom) (20). The DEBQ is a widely used questionnaire. These subscales have wellestablished internal consistency, and the restrained eating subscale has predictive validity for observed restrained eating $(20,21)$. The response options range from (1) never to (5) very often, and scores are averaged across subscales with higher scores indicating high levels of restrained, emotional, or external eating. In the current study, the DEBQ showed good internal reliability for restrained eating $(\alpha=0.91)$, external eating $(\alpha=0.82)$, and emotional eating $(\alpha=0.95)$.

Intuitive Eating Scale (IES): The IES was developed to measure adaptive eating behavior as an alternative to the typical focus on disordered eating (22). Recent research indicates that intuitive eating is a novel construct and not necessarily representative of disordered eating (23). 
Table 1 Characteristics of male and female subjects

\begin{tabular}{|c|c|c|}
\hline & $\begin{array}{l}\text { Female students } \\
\qquad(n=71)\end{array}$ & $\begin{array}{l}\text { Male students } \\
\quad(n=27)\end{array}$ \\
\hline Student age (years) & $18.5 \pm 0.95$ & $18.6 \pm 0.95$ \\
\hline Parent age (years) & $47.8 \pm 5.0$ & $48.0 \pm 4.9$ \\
\hline $\begin{array}{l}\text { Parent education (years } \\
\text { after age 16) }\end{array}$ & $5.3 \pm 2.4$ & $6.2 \pm 2.8$ \\
\hline Student BMI (kg/m²) & $24.2 \pm 5.3$ & $25.1 \pm 5.6$ \\
\hline Parent BMI (kg/m²) & $25.8 \pm 5.1$ & $23.9 \pm 4.4$ \\
\hline \multicolumn{3}{|l|}{ Parent-reported } \\
\hline Pressure & $2.2 \pm 1.0$ & $2.0 \pm 0.96$ \\
\hline Restriction & $2.6 \pm 0.87$ & $2.7 \pm 0.99$ \\
\hline Monitoring & $2.9 \pm 0.97$ & $3.2 \pm 0.75$ \\
\hline \multicolumn{3}{|l|}{ Student-reported } \\
\hline Pressure & $2.6 \pm 0.78$ & $2.9 \pm 0.67$ \\
\hline Restriction & $2.3 \pm 0.54$ & $2.5 \pm 0.54$ \\
\hline Monitoring & $2.9 \pm 1.3$ & $2.6 \pm 0.95$ \\
\hline \multicolumn{3}{|l|}{ DEBQ } \\
\hline Restrained eating & $2.8 \pm 0.85$ & $2.1 \pm 0.81^{*}$ \\
\hline External eating & $3.1 \pm 0.59$ & $3.0 \pm 0.55$ \\
\hline Emotional eating & $2.3 \pm 0.92$ & $2.1 \pm 0.95$ \\
\hline IES total score & $3.4 \pm 0.53$ & $3.8 \pm 0.65^{\star}$ \\
\hline Unconditional permission & $3.2 \pm 0.81$ & $3.7 \pm 0.90^{*}$ \\
\hline Physical reasons to eat & $3.3 \pm 0.82$ & $3.8 \pm 0.95^{\star}$ \\
\hline Hunger/satiety cues & $3.7 \pm 0.58$ & $3.9 \pm 0.56$ \\
\hline
\end{tabular}

Values are means \pm s.d.

DEBQ, Dutch Eating Behavior Questionnaire; IES, Intuitive Eating Scale.

'Differences between men and women ( $t$-test), $P<0.01$.

The IES consists of three subscales: assessing unconditional permission to eat, eating for physical reasons, and reliance on hunger/satiety cues. The IES can also be used to create a total intuitive eating score. The nineitem unconditional permission to eat subscale measures willingness to eat when physiologically hungry and what food is desired. The reliance on hunger/satiety cues subscale (six items) indicates awareness and use of hunger cues to determine when and how much to eat. The eatingfor-physical-reasons subscale (six items) assesses a willingness to eat to satisfy hunger rather than to eat for emotional reasons. The difference between the first two subscales is that one focuses on the awareness of hunger, whereas the other focuses on willingness to eat. This questionnaire has acceptable test-retest reliability, construct validity, and internal consistency values ranging from $\alpha=0.78$ to 0.85 . Higher scores on the IES indicate more positive eating habits. In the current study, the IES showed good internal reliability for unconditional eating $(\alpha=0.88)$, eating for physical reasons $(\alpha=0.87)$, and awareness of hunger and satiety cues $(\alpha=0.79)$.

Student BMI: Research assistants measured students' heights (using a standing stadiometer) and weights (using a Seca 882 BMI scale; Seca, Hanover, MD). Participants wore light clothing, removed their shoes, and were weighed and measured in triplicate to increase measurement reliability. Height and weight data were averaged and converted to BMI scores using the formula $\mathrm{kg} / \mathrm{m}^{2}$.

\section{Statistical analysis}

Independent sample $t$-tests showed that there were no significant differences between female $(n=71)$ and male $(n=27)$ participants with regard to demographic and anthropometric variables, so the sample
Table 2 Pearson's two-tailed correlations for parent- and student-reported child feeding practices

\begin{tabular}{lccc}
\hline \multicolumn{4}{c}{ Student retrospective reports } \\
\hline & Pressure & Restriction & Monitoring \\
\hline Parent retrospective reports & & & \\
Pressure & 0.14 & -0.14 & 0.12 \\
Restriction & 0.10 & 0.02 & 0.08 \\
Monitoring & 0.10 & 0.11 & $0.21^{*}$ \\
\hline${ }^{*} P<0.01$. & & &
\end{tabular}

was therefore collapsed across gender for the initial analysis and then divided by gender for the follow-up exploratory analysis. Pearson's two-tailed correlations were used to examine relationships between students' and their parents' retrospective reports of parental use of child feeding practices. Pearson correlations were also used to examine relationships between retrospective reports of child feeding practices and students' current eating behaviors and BMI. Independent sample $t$-tests were used to assess differences between parents' and students' reports of child feeding practices, between male and female students' reports, and between parents' reports of using feeding practices with daughters compared to sons. An $\alpha$ level of $P<0.01$ was adopted throughout the analyses to reduce the risk of type 1 errors.

\section{RESULTS \\ Participant characteristics}

The characteristics of the college students and their parents are summarized in Table 1. College students were 17-23 years old. Their reported ethnicity was $96 \%$ non-Hispanic white, $3 \%$ African American, and 1\% Asian American. Students' parents were 38-58 years old, they were mostly mothers (97\%), and most parents completed some formal schooling beyond secondary education. Mean BMIs for the two samples were 24.3 for the students (range 18.3-46.4) and 25.1 for parents (range 18.5-43.6). Thirty percent of male students were overweight and $11 \%$ were obese compared to $17 \%$ of overweight and $11 \%$ of obese female students. Male and female college students did not differ in their age or mean BMI, and their parents did not differ in their age, BMI, or educational level.

\section{College students' and their parents' recollections of child feeding practices}

Relationships between students' and parents' retrospective reports of child feeding practices are shown in Table 2. Students' recollections of their parents' use of restriction and pressure to eat were not correlated with their parents' recollections of these constructs; however, students' reports of parental monitoring were positively correlated to parents' recollections of their own monitoring.

\section{Relationships among retrospective CFQ, current eating behaviors, and BMI}

The results from Pearson's two-tailed correlations exploring the relationships between retrospective reports of child feeding practices, current eating behaviors, and BMI are provided in Table 3. Higher recollections of parental restriction and monitoring were positively correlated with higher student BMI and higher levels of emotional eating. Parental recollection of 
Table 3 Pearson's two-tailed correlations for parent-reported and student-reported child feeding practices, student-reported eating behavior, and measured student BMI

\begin{tabular}{|c|c|c|c|c|c|c|}
\hline & \multicolumn{3}{|c|}{ Student-reported CFQ } & \multicolumn{3}{|c|}{ Parent-reported CFQ } \\
\hline & Pressure & Restriction & Monitoring & Pressure & Restriction & Monitoring \\
\hline Student BMI & -0.07 & 0.19 & 0.11 & -0.14 & $0.36^{\star}$ & $0.27^{\star}$ \\
\hline Restrained eating & 0.03 & 0.05 & $0.25^{\star}$ & 0.10 & 0.15 & 0.09 \\
\hline \multicolumn{7}{|l|}{ Intuitive Eating Scale ${ }^{\mathrm{b}}$} \\
\hline $\begin{array}{l}\text { Unconditional } \\
\text { permission to eat }\end{array}$ & 0.04 & 0.10 & -0.16 & -0.05 & -0.06 & -0.08 \\
\hline $\begin{array}{l}\text { Eating for physical } \\
\text { reasons }\end{array}$ & 0.09 & 0.09 & -0.01 & -0.10 & -0.23 & $-0.27^{\star}$ \\
\hline
\end{tabular}

more monitoring was negatively correlated with eating for physical reasons on the IES. The only student-recalled feeding practice used by parents that was correlated with eating behaviors was parental monitoring, which was positively correlated with students' restrained eating behaviors.

\section{Gender differences in CFQ, eating behaviors, and current BMI relationships}

Table 4 provides additional information on these relationships when analyzed by gender. These results revealed relationships between parent-recollected, child feeding practices and current student eating behaviors and BMI for female students but not for male students in this sample. Specifically, parents who recollected more monitoring and restricting their daughters' food intake had daughters who reported significantly more emotional eating and had higher BMIs. In addition, higher levels of restrictive feeding practices used by parents were related to daughters' recollections of less eating for physical reasons of hunger and satiety. Parents who recollected more restriction of their son's food intake had sons with significantly heavier BMIs, but there were no other significant relationships between parental recollections of controlling feeding practices and eating behaviors in male participants.

\section{DISCUSSION}

This study examined links between student and parent recollections of early child feeding practices and current student eating behaviors and BMI. Using students' and parents' retrospective reports of child feeding practices that were used by parents when the college students were in middle childhood, we found significant positive correlations between the recollected use of controlling child feeding practices and current emotional eating in students. In addition, current BMI positively correlated with the parents' recollections of using controlling feeding practices in both male and female students. More relationships existed for parents' compared to students' recollections. The relationships between child feeding practices and eating behaviors were more apparent with females, even though there were no gender differences in BMI between men and women in this sample. Results from the current study provide evidence that gender needs to be considered when examining child feeding practices and may help understand previous contradictory findings in this field of research $(12,14-17)$.

Previous research focusing on parents' and children's perceptions of child feeding practices has shown that parents' and children's reports of parental use of pressure to eat are positively correlated, whereas their perceptions of parental use of restriction are often uncorrelated (8). In the current study, only children's and parents' recollections of monitoring were positively correlated. It is not a surprise that restriction was not correlated between student and parental reports because it is likely that some aspects of restrictive feeding practices occur beyond children's awareness if food is not being purchased, or it is being hidden or denied (24). Alternatively, it has been suggested that children are more consciously aware of being pressured to eat because this happens overtly. Family reports of monitoring have not been previously compared, but it is surprising that monitoring would be a feeding practice of which children are aware, and it may be that parents keep track of the foods their children eat by directly asking them. It is interesting that parent recollections, but not student recollections, were more strongly correlated to student eating behaviors and BMI, supporting previous work indicating that parental reports of their child feeding practices provide different information compared to child reports (25). These results conflict with an earlier finding that young girls' perceptions of parental pressure to eat, but not their parents' perceptions, were positively related to their own restrained and emotional eating (8). It may 
Table 4 Pearson's two-tailed correlations for parent-reported child feeding practices, student-reported eating behavior, and measured student BMI split by student gender

\begin{tabular}{|c|c|c|c|c|c|c|}
\hline & \multicolumn{3}{|c|}{ Female students $(n=71)$} & \multicolumn{3}{|c|}{ Male students $(n=27)$} \\
\hline & Pressure & Restriction & Monitoring & Pressure & Restriction & Monitoring \\
\hline Student BMI & -0.07 & $0.31^{*}$ & $0.29^{\star}$ & -0.30 & $0.47^{\star}$ & 0.16 \\
\hline Restrained eating & 0.14 & 0.19 & 0.22 & -0.13 & 0.13 & -0.12 \\
\hline External eating & 0.17 & 0.16 & 0.19 & -0.31 & 0.13 & 0.12 \\
\hline \multicolumn{7}{|l|}{ Intuitive Eating Scale ${ }^{b}$} \\
\hline $\begin{array}{l}\text { Unconditional } \\
\text { permission to eat }\end{array}$ & -0.08 & -0.05 & -0.19 & 0.13 & -0.12 & 0.10 \\
\hline $\begin{array}{l}\text { Eating for physical } \\
\text { reasons }\end{array}$ & -0.13 & $-0.33^{*}$ & $-0.40^{*}$ & 0.06 & -0.07 & -0.07 \\
\hline
\end{tabular}

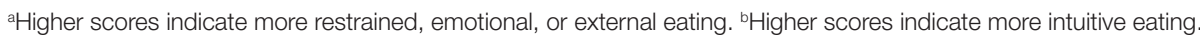

${ }^{*} P<0.01$.

be that parents' and children's perceptions, and understanding of child feeding practices are dynamic over the course of childhood and adolescence.

Results from this study are unique because of the relationship between parental reports of their early child feeding practices and current eating behavior and BMI in young adults. That parental retrospective reports of their early child feeding practices correlated primarily to current emotional eating in females is intriguing, especially given that there were no mean differences in reported child feeding practices between genders and in both genders, restrictive feeding practices were related to BMI. Given the borderline significance for some of the values with male participants, the results would have mostly likely differed with a larger male sample and implied a different pattern of relationships for male students compared to female students. It is unclear why emotional eating was the only construct related to the parents' recollections of restriction and monitoring. In a recent study, depression was related to emotional eating but not external eating, implying that there are likely other psychological factors involved in the complexity of eating behavior (26). The relationships seen between emotional eating, and the use of restriction and monitoring in this study are troubling given that emotional eating may be less treatable than other types of maladaptive eating behaviors (27).

Recently, an observational study of family eating interactions revealed that girls more readily complied with parental demands concerning food consumption compared to boys and suggested that girls may be more sensitive to parental requests than boys (15). Perhaps expectations about the body image of girls make them more vulnerable to the effects of controlling child feeding practices. If parents' recollections are more of a reflection of current eating behaviors in their daughters rather than an influence, it is interesting that the relationship exists for female but not male students. In a another study using a sibling design, parents reported using different feeding practices that mirrored differences in eating behaviors in the siblings (28).

Due to the retrospective design of this study, it is not possible to know whether parents' reports of controlling feeding practices are a reaction to, or a predictor of, the students' current eating behaviors. Nevertheless, these results suggest a need for continued research in understanding how parent-child interactions concerning food might influence the development of eating behaviors. Although the use of retrospective reports revealed compelling relationships between feeding practices and emotional eating, the validity of using retrospective reports for this purpose is untested. It is possible that participants' recollections of past feeding behaviors were not precise. Moreover, they may have been biased by their adult child's current eating behavior and weight status. Future research will want to explore the directionality of these relationships. Links between child feeding practices and eating behaviors may be different for various ethnic or socioeconomic groups (10); thus, the findings from this homogeneous sample are limited in their generalizability. In addition, the unequal sample size of male and females limits our ability to make conclusions about how gender differences relate to the use of child feeding practices by parents, and to the development of eating behaviors and weight status in college students.

Findings from this study provide insight about child feeding practices from the perspective of parents and their collegeaged children. The approach used in this research was novel in that retrospective reports were used to examine relationships between early child feeding practices and current eating behaviors and BMI in adulthood. These findings indicate that restrictive and monitoring feeding practices recollected by parents have potentially long-term consequences, perhaps contributing to the development of emotional eating in female grown children. The results also suggest that retrospective reports from parents, but not students, may be more informative in 
understanding links to the development of eating behaviors and BMI in early adulthood. These data indicate that past inconsistencies in the literature may be age or gender related, and that the relationship among child feeding practices, eating behavior, and BMI might become more evident as girls reach adulthood.

\section{DISCLOSURE}

The authors declared no conflict of interest.

\section{REFERENCES}

1. Batsell WR Jr, Brown AS, Ansfield ME, Paschall GY. "You will eat all of that!": a retrospective analysis of forced consumption episodes. Appetite 2002;38:211-219.

2. Faith MS, Berkowitz RI, Stallings VA et al. Parental feeding attitudes and styles and child body mass index: prospective analysis of a gene-environment interaction. Pediatrics 2004;114:e429-e436.

3. Farrow CV, Blissett J. Controlling feeding practices: cause or consequence of early child weight? Pediatrics 2008;121: e164-e169.

4. Ventura AK, Birch LL. Does parenting affect children's eating and weight status? Int J Behav Nutr Phys Act 2008;5:15.

5. Constanza PR, Woody EZ. Domain-specific parenting styles and their impact on the child's development of particular deviance: the example of obesity proneness. J Soc Clin Psychol 1985;3:425.

6. Birch LL, Fisher JO, Davison KK. Learning to overeat: maternal use of restrictive feeding practices promotes girls' eating in the absence of hunger. Am J Clin Nutr 2003;78:215-220.

7. Galloway AT, Fiorito LM, Francis LA, Birch LL. 'Finish your soup': counterproductive effects of pressuring children to eat on intake and affect. Appetite 2006;46:318-323.

8. Carper JL, Orlet Fisher J, Birch LL. Young girls' emerging dietary restraint and disinhibition are related to parental control in child feeding. Appetite 2000;35:121-129.

9. Carnell S, Wardle J. Associations between multiple measures of parental feeding and children's adiposity in United Kingdom preschoolers. Obesity (Silver Spring) 2007;15:137-144.

10. Corsini N, Danthiir V, Kettler L, Wilson C. Factor structure and psychometric properties of the Child Feeding Questionnaire in Australian preschoo children. Appetite 2008:51:474-481.

11. Kaur $\mathrm{H}$, Li C, Nazir $\mathrm{N}$ et al. Confirmatory factor analysis of the child-feeding questionnaire among parents of adolescents. Appetite 2006;47:36-45.

12. Snoek HM, Engels RC, Janssens JM, van Strien T. Parental behaviour and adolescents' emotional eating. Appetite 2007;49:223-230.
13. Strauss RS. Self-reported weight status and dieting in a cross-sectional sample of young adolescents: National Health and Nutrition Examination Survey III. Arch Pediatr Adolesc Med 1999;153:741-747.

14. Johnson SL, Birch LL. Parents' and children's adiposity and eating style. Pediatrics 1994:94:653-661.

15. Orrell-Valente JK, Hill LG, Brechwald WA et al. "Just three more bites": an observational analysis of parents' socialization of children's eating at mealtime. Appetite 2007;48:37-45.

16. Fisher JO, Birch LL. Restricting access to foods and children's eating. Appetite 1999;32:405-419.

17. Blissett J, Meyer C, Haycraft E. Maternal and paternal controlling feeding practices with male and female children. Appetite 2006;47: 212-219.

18. McKinney C, Renk K. Differential parenting between mothers and fathers: implications for late adolescents. J Fam Issues 2008;29: 806-827.

19. Birch LL, Fisher JO, Grimm-Thomas K et al. Confirmatory factor analysis of the Child Feeding Questionnaire: a measure of parental attitudes, beliefs and practices about child feeding and obesity proneness. Appetite 2001;36:201-210.

20. Van Strien T, Frijters JE, Bergers JP, Defares PB. The Dutch Eating Behavior Questionnaire (DEBQ) for assessment of restrained, emotional, and external eating behavior. Int J Eat Disord 1986;5:295-315.

21. Van Strien T, Frijters JE, Van Staveren WA, Defares PB, Deurenberg P. The predictive validity of the Dutch Restrained Eating Scale. Int J Eat Disord 1986;5:747-755

22. Tylka TL. Development and psychometric evaluation of a measure of intuitive eating. J Couns Psychol 2006;53:226-240.

23. Tylka TL, Wilcox J. Are intuitive eating and eating disorder symptomatology opposite poles of the same construct? J Couns Psychol 2006;53: 474-485.

24. Ogden J, Reynolds R, Smith A. Expanding the concept of parental control: a role for overt and covert control in children's snacking behaviour? Appetite 2006;47:100-106.

25. Haines J, Neumark-Sztainer D, Hannan P, Robinson-O'Brien R. Child versus parent report of parental influences on children's weight-related attitudes and behaviors. J Pediatr Psychol 2008;33:783-788.

26. Ouwens MA, van Strien T, van Leeuwe JF. Possible pathways between depression, emotional and external eating. A structural equation model. Appetite 2009;53:245-248.

27. Van Strien T, Van de Laar FA. Intake of energy is best predicted by overeating tendency and consumption of fat is best predicted by dietary restraint: a 4-year follow-up of patients with newly diagnosed type 2 diabetes. Appetite 2008;50:544-547.

28. Farrow CV, Galloway AT, Fraser K. Sibling eating behaviours and differential child feeding practices reported by parents. Appetite 2009:52:307-312. 